\title{
Generalized Kraus Operators and Generators of Quantum Dynamical Semigroups
}

\author{
Sabina Alazzawi ${ }^{1}$, Bernhard Baumgartner ${ }^{2}$ \\ Fakultät für Physik, Universität Wien \\ Boltzmanngasse 5, A-1090 Vienna, Austria
}

Dezember, 2013

\begin{abstract}
Quantum dynamical semigroups play an important role in the description of physical processes such as diffusion, radiative decay or other nonequilibrium events. Taking strongly continuous and trace preserving semigroups into consideration, we show that, under a special criterion, the generator of such a group admits a certain generalized standard form, thereby shedding new light on known approaches in this direction. Furthermore, we illustrate our analysis in concrete examples.
\end{abstract}

Keywords: open system, time evolution, Lindblad generator, semigroup

PACS numbers: $\quad$ 03.65.Yz,$\quad$ 05.40.-a , 42.50.Dv , 03.65.Fd

\footnotetext{
${ }^{1}$ sabina.alazzawi@univie.ac.at

${ }^{2}$ Bernhard.Baumgartner@univie.ac .at
} 


\section{Introduction}

Time evolutions of open quantum systems are not appearing as unitary maps. Phenomenological equations describing non-equilibrium processes, like diffusion, radiative decay, radiative excitation, etc., have no terms describing memoryeffects, they are Markovian. These equations show the arrow of time, one cannot follow evolutions indefinitely backwards, so they are to be described with semigroups of maps $\mathcal{T}^{t}$, acting on density matrices.

Basic dynamics is reversible. In the quest for answering the question of how irreversibility can emerge one has to perform limiting processes. The methods of deriving $\mathcal{T}^{t}$ from basic unitary dynamics, see [D74, D76, D76b], using weak coupling or singular coupling limits, give rise to semigroups of completely positive maps.

The generally possible structure of generators of such quantum dynamical processes has been completely characterized for norm-continuous semigroups, yielding the "GKS-Lindblad-equation", GKS76, L76]. Further attempts to study strongly continuous evolutions by E.B.Davies, [D77, D77b, D80], led, with some special assumptions, to a "standard form" of generators. A.S.Holevo extended this study further, [K95, H96, H97. He defines a generator in the Heisenberg picture as a quadratic form on the Hilbert space. Crucial results of investigations in this sense are presented by F.Fagnola and R.Rebolledo in FR06, sect. 3.

In this paper we rework their methods. We define a generalized standard form for each generator of a QDS (quantum dynamical semigroup which is strongly continuous) in the Schrödinger picture and from a purely functional analytic point of view. It allows for a complete characterization in case the QDS is unambiguously defined by its "matrix normal" action on the matrix units in some basis of the Hilbert space. Two new examples of a QDS are presented, showing all the special features of the present analysis. One is as simple and clear as possible, the other one models a natural process observed in modern quantum physics.

\section{Setup and main statements}

We use the known fact that each strongly continuous semigroup on a Banach space has a generator $\mathcal{L}$, a closed operator with dense domain of definition $\mathscr{D}(\mathcal{L})$ which is the set of all those Banach space elements $\rho$ for which the limit $t \rightarrow 0$ in the formula defining $\mathcal{L}$,

$$
\mathcal{L}(\rho)=\lim _{t \rightarrow 0} \frac{1}{t}\left(\mathcal{T}^{t}(\rho)-\rho\right)
$$

exists in norm. (In [D76b] such a semigroup is denoted as " $c_{0}$ semigroup".) Now a QDS acts on three levels: Hilbert space, density matrices, "super operators" 
acting on density matrices. The goal is to represent the generator $\mathcal{L}$ by way of decomposing its action into actions described by Hilbert space operators. Under certain assumptions stated below, this can be done by considering

- a separable Hilbert space $\mathscr{H}$,

- the Banach space of trace class operators $\mathfrak{T}(\mathscr{H})$ with the trace norm,

- a strongly continuous semigroup of trace preserving completely positive maps $\mathcal{T}^{t}: \mathfrak{T}(\mathscr{H}) \rightarrow \mathfrak{T}(\mathscr{H})$ with generator $\mathcal{L}$.

\section{DEFINITION. Generalized standard form}

Consider $L_{k}$ and $M$, linear operators on $\mathscr{H}$ with dense domains of definition, and with the properties that $M$ is closed and generates a semigroup on $\mathscr{H}$, and each $L_{k}$ is relatively bounded with respect to $M$. Consider, moreover, "generalized operators" $L_{k}^{\dagger}$ and $M^{\dagger}$, linear operators mapping $\mathscr{H}$ into the conjugate algebraic dual of $\mathscr{D}(M)$, such that for all $\psi \in \mathscr{H}$ and for all $\eta \in \mathscr{D}(M)$,

$$
\left\langle\eta\left|L_{k}^{\dagger}\right| \psi\right\rangle:=\left\langle\psi\left|L_{k}\right| \eta\right\rangle^{*}, \quad\left\langle\eta\left|M^{\dagger}\right| \psi\right\rangle:=\langle\psi|M| \eta\rangle^{*} .
$$

Then the generator $\mathcal{L}$ of a trace preserving $Q D S$ on $\mathfrak{T}(\mathscr{H})$ is in generalized standard form if

$$
\begin{aligned}
\mathcal{L}(\rho)= & \sum_{k} L_{k} \cdot \rho \cdot L_{k}^{\dagger}-M \cdot \rho-\rho \cdot M^{\dagger}, \\
& \sum_{k} L_{k}^{\dagger} L_{k}=M+M^{\dagger} .
\end{aligned}
$$

Equation (3) holds for $\rho$ of finite rank which can be represented as finite sums of dyadic products $\sum_{n}\left|\phi_{n}\right\rangle\left\langle\psi_{n}\right|$ with $\left\{\phi_{n}, \psi_{n}\right\} \subset \mathscr{D}(M)$. The domain $\mathscr{D}(\mathcal{L})$ is the closure of this set $\mathscr{D}_{0}$ in graph norm $\|\rho\|_{\mathcal{L}}=\|\rho\|_{1}+\|\mathcal{L}(\rho)\|_{1}$. Equation (4) holds for the noted entities as quadratic forms with $\mathscr{D}(M)$ as the common form domain.

In sloppy words, the operators $M^{\dagger}$ and $L_{k}^{\dagger}$ have their domain of definition at the left hand side. In Section 4 we discuss these notations and the relation to Davies' investigations.

To prove the existence of such a generalized standard form we need the assumption that there is some basis, in which the matrix elements of density operators are differentiable functions of time. We, therefore, consider:

- a basis $\left\{e_{k}\right\} \subset \mathscr{H}$ and the dense set of finite linear combinations of these basis elements

$$
\mathscr{D}_{e}:=\operatorname{lin}\left\{e_{k}\right\},
$$

- the set of finite linear combinations of matrix units in this basis

$$
\mathscr{D}_{e}^{2}:=\operatorname{lin}\left\{\left|e_{k}\right\rangle\left\langle e_{\ell}\right|\right\} \subset \mathfrak{T}(\mathscr{H}) .
$$




\section{DEFINITION. Matrix normal $Q D S$}

A QDS on $\mathfrak{T}(\mathscr{H})$ is called "matrix normal" if there exists a basis $\left\{e_{k}\right\}$ of $\mathscr{H}$ such that $\mathscr{D}_{e}^{2}$ as defined in (b) is a core for its generator $\mathcal{L}$.

The operator $\mathcal{L}$ is then completely defined by its action on each $\rho$ in the core $\mathscr{D}_{e}^{2}$. It is hence decomposable into its actions on the matrix units $\left|e_{k}\right\rangle\left\langle e_{\ell}\right|$ in the basis $\left\{e_{k}\right\}$. It is, for our intentions, not sufficient to assume nothing but that $\mathscr{D}_{e}^{2}$ is just contained in the domain of definition of $\mathcal{L}$. In Section 4 we explain this remark and give an example.

This formal assumption on decomposability with respect to matrix elements is now related to the description of generators $\mathcal{L}$ in the generalized standard form, using operators on the underlying Hilbert space $\mathscr{H}$, without a priori reference to a basis. The main result of the present investigation is:

3 THEOREM. Matrix normality is equivalent to the existence of a generalized standard form.

There exists a generalized standard form for the generator $\mathcal{L}$ of a trace preserving $Q D S$ if and only if it is matrix normal. In this case $\mathscr{D}_{e} \subset \mathscr{D}(M)$.

\section{Proofs and further details}

\subsection{From decomposability to the standard form.}

Before we come to the proof of Theorem [3, some preparation is necessary. We start by stating the following

4 LEMMA. The generator $\mathcal{L}$ of a matrix normal trace preserving $Q D S$ has the properties:

1. $\forall \phi \in \mathscr{D}_{e}: \quad\langle\phi|\mathcal{L}(|\phi\rangle\langle\phi|)| \phi\rangle \leq 0$,

2. $\forall \phi \in \mathscr{D}_{e}, \forall \psi$ with $\langle\phi \mid \psi\rangle=0: \quad\langle\psi|\mathcal{L}(|\phi\rangle\langle\phi|)| \psi\rangle \geq 0$,

3. $\forall\left\{\phi_{k} \in \mathscr{D}_{e}, \psi_{k}\right\}$ with $\sum_{k}\left\langle\phi_{k} \mid \psi_{k}\right\rangle=0: \quad \sum_{k, \ell}\left\langle\psi_{k}\left|\mathcal{L}\left(\left|\phi_{k}\right\rangle\left\langle\phi_{\ell}\right|\right)\right| \psi_{\ell}\right\rangle \geq 0$.

Proof. Item (1) is a consequence of preservation of both positivity and trace by transformation of $|\phi\rangle\langle\phi|$ by $\mathcal{T}^{t}$. Starting at $t=0$ with $\rho=|\phi\rangle\langle\phi|$, some new positive diagonal elements may appear in $\mathcal{T}^{t}(|\phi\rangle\langle\phi|)$. Since the sum of all the diagonal elements is constant, the original single element, which was "1", when $\|\phi\|=1$, has to diminish. Item (2) concerns the newly appearing diagonal elements as $t$ increases, starting from zero, and follows again from positivity of $\mathcal{T}^{t}$. Item (3) follows from complete positivity of $\mathcal{T}^{t}$. It is item (2) applied to the generator $\hat{\mathcal{L}}$ of the semigroup $\hat{\mathcal{T}}^{t}=\mathcal{T}^{t} \otimes \mathrm{Id}$, acting on density matrices on $\mathscr{H} \otimes \ell^{2}$, where Id is the identity-map, with $\hat{\phi}=\sum_{k} \phi_{k} \otimes f_{k}, \hat{\psi}=\sum_{k} \psi_{k} \otimes f_{k}$, where $\left\{f_{k}\right\}$ is an orthonormal basis of $\ell^{2}$. 
The subsequent construction follows Holevo [H97]; (see also, for the case of norm continuous semigroups, [F99]; the method, according to F.Fagnola, has already been used earlier by A.Frigerio):

5 DEFINITION. The generator of contraction in Hilbert space, $M$ For a matrix normal $Q D S$ choose some vector $\chi \in \mathscr{D}_{e}$ with $\|\chi\|=1$. On $\mathscr{D}(M):=$ $\{\psi:|\psi\rangle\langle\chi| \in \mathscr{D}(\mathcal{L})\}$ an operator $M$ is defined by

$$
M \psi:=-\mathcal{L}(|\psi\rangle\langle\chi|) \chi+\frac{1}{2}\langle\chi|\mathcal{L}(|\chi\rangle\langle\chi|)| \chi\rangle \psi
$$

The naming is explained in Proposition 8 . Note that $\mathscr{D}_{e} \subset \mathscr{D}(M)$, which will be used in Equation (8).

6 PROPOSITION. The expander in the generator of the $Q D S$ For a matrix normal QDS the map

$$
\mathcal{L}_{+}: \rho \mapsto \mathcal{L}(\rho)+M \rho+\rho M^{\dagger}
$$

is a completely positive map from $\mathscr{D}_{e}^{2}$ into $\mathfrak{T}(\mathscr{H})$.

Proof. Each $\sigma \in \mathscr{D}_{e}^{2}$ can be expanded as a linear combination of at most four positive matrices of finite rank. Each positive matrix $\rho \in \mathscr{D}_{e}^{2}$ can be represented as $\rho=\sum_{n=1}^{N}\left|e_{n}\right\rangle\left\langle e_{n}\right|$ for some elements $e_{n} \in \mathscr{D}_{e}$, and there exists

$$
\operatorname{Tr}\left\{M \rho+\rho M^{\dagger}\right\}=\sum_{n=1}^{N}\left\langle e_{n}\left|\left(M+M^{\dagger}\right)\right| e_{n}\right\rangle<\infty .
$$

Now $\mathcal{L}$ generates a semigroup which preserves the trace, so for all $\rho \in \mathscr{D}(\mathcal{L})$

$$
\operatorname{Tr}\{\mathcal{L}(\rho)\}=\left[\frac{d}{d t} \operatorname{Tr}\left\{\mathcal{T}^{t}(\rho)\right\}\right]_{t=0}=0,
$$

and this implies, for all $\rho \in \mathscr{D}_{e}^{2}$

$$
\operatorname{Tr}\left\{\mathcal{L}_{+}(\rho)\right\}=\operatorname{Tr}\left\{M \rho+\rho M^{\dagger}\right\}
$$

and $\mathcal{L}_{+}$is well defined on $\mathscr{D}_{e}^{2}$.

The criterion for complete positivity states that $\sum_{k, \ell}\left\langle\psi_{k}\left|\mathcal{L}_{+}\left(\left|\phi_{k}\right\rangle\left\langle\phi_{\ell}\right|\right)\right| \psi_{\ell}\right\rangle$ is non-negative for all $N$ and $\left\{\left(\phi_{k}, \psi_{k}\right), k=1 \ldots N, \phi_{k} \in \mathscr{D}_{e}\right\}$. To check whether this criterion is fulfilled, we insert (77) into (8) $)$. We get

$$
\begin{aligned}
\left\langle\psi_{k}\left|\mathcal{L}_{+}\left(\left|\phi_{k}\right\rangle\left\langle\phi_{\ell}\right|\right)\right| \psi_{\ell}\right\rangle= & \\
\left\langle\psi_{k}\left|\mathcal{L}\left(\left|\phi_{k}\right\rangle\left\langle\phi_{\ell}\right|\right)\right| \psi_{\ell}\right\rangle & +\langle\chi|\mathcal{L}(|\chi\rangle\langle\chi|)| \chi\rangle \cdot\left\langle\psi_{k} \mid \phi_{k}\right\rangle \cdot\left\langle\phi_{\ell} \mid \psi_{\ell}\right\rangle \\
-\left\langle\psi_{k}\left|\mathcal{L}\left(\left|\phi_{k}\right\rangle\langle\chi|\right)\right| \chi\right\rangle \cdot\left\langle\phi_{\ell} \mid \psi_{\ell}\right\rangle & -\left\langle\chi\left|\mathcal{L}\left(|\chi\rangle\left\langle\phi_{\ell}\right|\right)\right| \psi_{\ell}\right\rangle \cdot\left\langle\psi_{k} \mid \phi_{k}\right\rangle .
\end{aligned}
$$

Defining

$$
\phi_{N+k}:=-\left\langle\psi_{k} \mid \phi_{k}\right\rangle \cdot \chi, \quad \psi_{N+k}:=\chi
$$


we continue the reformulation of (11), writing it as

$$
\sum_{i=0}^{1} \sum_{j=0}^{1}\left\langle\psi_{k+i N}\left|\mathcal{L}\left(\left|\phi_{k+i N}\right\rangle\left\langle\phi_{\ell+j N}\right|\right)\right| \psi_{\ell+j N}\right\rangle .
$$

Summation over all indices gives

$$
\sum_{k=1}^{N} \sum_{\ell=1}^{N}\left\langle\psi_{k}\left|\mathcal{L}_{+}\left(\left|\phi_{k}\right\rangle\left\langle\phi_{\ell}\right|\right)\right| \psi_{\ell}\right\rangle=\sum_{k=1}^{2 N} \sum_{\ell=1}^{2 N}\left\langle\psi_{k}\left|\mathcal{L}\left(\left|\phi_{k}\right\rangle\left\langle\phi_{\ell}\right|\right)\right| \psi_{\ell}\right\rangle \geq 0 .
$$

The inequality at the end holds because of the property stated in Lemma (4,3) of $\mathcal{L}$ and $\sum_{k=1}^{2 N}\left\langle\phi_{k} \mid \psi_{k}\right\rangle=0$, which follows from (12). Complete positivity of $\mathcal{L}_{+}$ is thus established.

With these preparations we now come to the proof of the algebraic part of Theorem 3 :

\section{Proof. Algebraic skeleton of the extended standard form:}

Due to Proposition 6, Theorem 14] and Corollary 16, given in the Appendix in Section 6, we have for all $\rho \in \mathscr{D}_{e}^{2}$ that $\mathcal{L}(\rho)$ can be decomposed as

$$
\mathcal{L}(\rho)=\mathcal{L}_{+}(\rho)-M \rho-\rho M^{\dagger}
$$

Moreover, there exist generalized Kraus Operators $L_{k}$, acting on $\mathscr{D}_{e}$, such that

$$
\mathcal{L}_{+}(\rho)=\sum_{k} L_{k} \cdot \rho \cdot L_{k}^{\dagger}
$$

Proof of Equation (4):

Operations which are standard procedures in dealing with ordinary operators have to be carefully adapted to the case where generalized operators appear. Consider $\rho=|\phi\rangle\langle\phi| \in \mathscr{D}_{e}^{2}$ with $\|\phi\|=1$. So $\mathcal{L}(\rho) \in \mathfrak{T}(\mathscr{H})$ and $\mathcal{T}^{t}(\rho)$ is differentiable:

$$
\begin{gathered}
0=\left.\frac{d}{d t} \operatorname{Tr}\left(\mathcal{T}^{t}(\rho)\right)\right|_{t=0}=\operatorname{Tr}(\mathcal{L}(\rho))=\sum_{\alpha}\left\langle\alpha\left|\left(\sum_{k} L_{k} \rho L_{k}^{\dagger}-M \rho-\rho M^{\dagger}\right)\right| \alpha\right\rangle= \\
=\sum_{\alpha}\left(\sum_{k}\left\langle\alpha\left|L_{k}\right| \phi\right\rangle\left\langle\phi\left|L_{k}^{\dagger}\right| \alpha\right\rangle-\langle\alpha|M| \phi\rangle\langle\phi \mid \alpha\rangle-\langle\alpha \mid \phi\rangle\left\langle\phi\left|M^{\dagger}\right| \alpha\right\rangle\right),
\end{gathered}
$$

for any basis $\alpha$. We choose a basis with $|\alpha=1\rangle=|\phi\rangle$, so $\langle\alpha \mid \phi\rangle=\delta_{\alpha, 1}$, and

$$
\operatorname{Tr}(\mathcal{L}(\rho))=\sum_{\alpha}\left(\sum_{k}\left|\left\langle\alpha\left|L_{k}\right| \phi\right\rangle\right|^{2}-\left(\langle\alpha|M| \phi\rangle+\left\langle\phi\left|M^{\dagger}\right| \alpha\right\rangle\right) \delta_{\alpha, 1}\right)=
$$




$$
=\sum_{\alpha}\left(\sum_{k}\left|\left\langle\alpha\left|L_{k}\right| \phi\right\rangle\right|^{2}\right)-\left\langle\phi\left|\left(M+M^{\dagger}\right)\right| \phi\right\rangle .
$$

Since $\operatorname{Tr}(\mathcal{L}(\rho))=0$ and the expectation value of $M$ is finite, the first term with the double sum is also finite. This sum over non-negative numbers, however, can be rearranged as follows:

$$
\sum_{\alpha} \sum_{k}\left|\left\langle\alpha\left|L_{k}\right| \phi\right\rangle\right|^{2}=\sum_{k} \sum_{\alpha}\left\langle\phi\left|L_{k}^{\dagger}\right| \alpha\right\rangle\left\langle\alpha\left|L_{k}\right| \phi\right\rangle=\left\langle\phi\left|\sum_{k} L_{k}^{\dagger} L_{k}\right| \phi\right\rangle .
$$

Therefore, the formula

$$
\left\langle\phi\left|\left(\sum_{k} L_{k}^{\dagger} L_{k}-M-M^{\dagger}\right)\right| \phi\right\rangle=0
$$

holds for each vector $\phi \in \mathscr{D}_{e}$, and Equation (44) is proven to be valid on the restricted form domain $\mathscr{D}_{e}$.

Having built the algebraic structure, we consider analytic-topological features. From Equation (4) on its skeleton domain it follows immediately that each $L_{k}$ is bounded relative to $M$, that is

$$
\left\|L_{k} \phi\right\|^{2}=2 \operatorname{Re}\langle\phi|M| \phi\rangle \leq 2\|\phi\| \cdot\|M \phi\|<(\|\phi\|+\|M \phi\|)^{2} .
$$

The $L_{k}$ can therefore be uniquely extended to the entire domain $\mathscr{D}(M)$. The super operator $\mathcal{L}_{+}$and the validity of Equation (3) can be extended to $\mathscr{D}_{0}$, and Equation (4) holds on the whole form domain.

In order to finish the proof of one half of Theorem [3, our last task is to show that $M$ is closed and generates a contraction semigroup.

\section{PROPOSITION. $M$ is closed.}

The operator $M$, the mover-and-contractor presented in Definition 5, is closed.

Proof. We consider a sequence $\psi_{n} \in \mathscr{D}(M)$, with $\psi_{n} \rightarrow \psi$ and $M \psi_{n} \rightarrow \eta$. With $\psi_{m, n}:=\psi_{m}-\psi_{n}$, using Equation (10), we have

$$
\begin{gathered}
0 \leq \operatorname{Tr}\left\{\mathcal{L}_{+}\left(\left|\psi_{m, n}\right\rangle\left\langle\psi_{m, n}\right|\right)\right\}=\operatorname{Tr}\left\{M\left|\psi_{m, n}\right\rangle\left\langle\psi_{m, n}|+| \psi_{m, n}\right\rangle\left\langle\psi_{m, n}\right| M^{\dagger}\right\} \\
\leq 2 \cdot\left\|\psi_{m}-\psi_{n}\right\| \cdot\left\|M\left(\psi_{m}-\psi_{n}\right)\right\| \rightarrow 0
\end{gathered}
$$

as $m, n \rightarrow \infty$. All following limits of operators are now existing in trace norm.

For any $\gamma \in \mathscr{D}_{e}$, any $z \in \mathbb{C}$, we have

$$
\begin{gathered}
0 \leq \mathcal{L}_{+}\left(\left|\gamma+z \psi_{m, n}\right\rangle\left\langle\gamma+z \psi_{m, n}\right|\right)= \\
=\mathcal{L}_{+}(|\gamma\rangle\langle\gamma|)+z \mathcal{L}_{+}\left(\left|\psi_{m, n}\right\rangle\langle\gamma|\right)+z^{*} \mathcal{L}_{+}\left(|\gamma\rangle\left\langle\psi_{m, n}\right|\right)+|z|^{2} \mathcal{L}_{+}\left(\left|\psi_{m, n}\right\rangle\left\langle\psi_{m, n}\right|\right)
\end{gathered}
$$


The first term is constant and the last one vanishes in the limit $m, n \rightarrow \infty$ for arbitrary $|z|$. The two terms in between, depending linearly on $z$ and being negative for some argument of $z$, have to remain bounded in relation to $\mathcal{L}_{+}(|\gamma\rangle\langle\gamma|)$, independently of $z$, hence each one has to go to zero, especially $\mathcal{L}_{+}\left(\left|\psi_{m, n}\right\rangle\langle\gamma|\right) \rightarrow 0$.

It follows that $\mathcal{L}_{+}\left(\left|\psi_{n}\right\rangle\langle\gamma|\right)$ is a Cauchy sequence, and therefore also $\mathcal{L}\left(\left|\psi_{n}\right\rangle\langle\gamma|\right)$ converges. Since $\mathcal{L}$ is closed, $|\psi\rangle\langle\gamma| \in \mathscr{D}(\mathcal{L})$ for each $\gamma \in \mathscr{D}_{e}$ and hence $\psi \in \mathscr{D}(M)$ and $M \psi=\eta$, by definition.

\section{PROPOSITION. $M$ generates a semigroup on the Hilbert space.}

The operator $M$ is maximal accretive. It generates the contractive semigroup $e^{-t M}$ on the Hilbert space $\mathscr{H}$.

Proof. $M$ is accretive ( $-M$ is dissipative), i.e. $\operatorname{Re}\langle\phi|M| \phi\rangle \geq 0 \forall \phi \in \mathscr{D}(M)$, as is seen in Equation 4. It generates a semigroup iff it is maximal accretive.

Suppose now that it is not maximal and that there exists an accretive extension $\bar{M}$, being maximal and hence generating a semigroup. In its domain $\mathscr{D}(\bar{M})$, considered as a Hilbert space with norm (squared) $\|\phi\|_{\bar{M}}^{2}=\|\bar{M} \phi\|^{2}+\|\phi\|^{2}$, there exists a non-empty subspace $\overline{\mathscr{D}}$ orthogonal to $\mathscr{D}(M)$. Extend further the operators $L_{k}$ from acting on $\mathscr{D}(M)$ to acting on $\mathscr{D}(\bar{M})$ by $L_{k} \phi=0$ for all $\phi \in \overline{\mathscr{D}}$. Then the inequality $\sum_{k} L_{k}^{\dagger} L_{k} \leq \bar{M}+\bar{M}^{\dagger}$ holds. Therefore, under these conditions there exists a contractive semigroup on $\mathfrak{T}(\mathscr{H})$ with generator $\overline{\mathscr{L}}$ such that

$$
\overline{\mathscr{L}}(|\phi\rangle\langle\psi|)=\sum_{k}\left|L_{k} \phi\right\rangle\left\langle L_{k} \psi|-| \bar{M} \phi\right\rangle\langle\psi|-| \phi\rangle\langle\bar{M} \psi|, \quad \forall(\phi, \psi) \subset \mathscr{D}(\bar{M}),
$$

as proven in [F99, Section 3]. However, such an $\overline{\mathscr{L}}$ would be an extension of $\mathscr{L}$, a contradiction to the maximal dissipativity of generators of semigroups.

The properties of $M$ and of the $L_{k}$ are in precise correlation to the structure of generators of QMS as defined in [F99] and [FR06].

\subsection{Deriving decomposability from the standard form}

We assume the existence of a generator $\mathcal{L}$ in generalized standard form and we divide it, as above, into two pieces. One of it is the completely positive map $\mathcal{L}_{+}$, the other one shall now be investigated in more detail.

9 DEFINITION. On the Banach space $\mathfrak{T}(\mathscr{H})$ we consider the operator

$$
\mathcal{M}: \rho \mapsto M \rho+\rho M^{\dagger}
$$

with domain $\mathscr{D}_{0}$, as defined in Definition 1 as a subset of $\mathscr{D}(\mathcal{L})$, namely

$$
\mathscr{D}_{0}:=\left\{\rho=\sum_{n=1}^{N}\left|\phi_{n}\right\rangle\left\langle\psi_{n}\right|,\left\{\phi_{n}, \psi_{n}\right\} \in \mathscr{D}(M), N<\infty\right\} .
$$


It follows that also $\mathcal{L}_{+}=\mathcal{L}+\mathcal{M}$ is well defined on $\mathscr{D}_{0}$. Note that the expander $\mathcal{L}_{+}$is dominated by $\mathcal{M}$. Namely, as in the proof of Proposition 6, Equation (9), we have the general condition that $\mathcal{L}$ generates a semigroup which preserves the trace, hence for all $\rho \in \mathscr{D}(\mathcal{L})$

$$
\operatorname{Tr}\{\mathcal{L}(\rho)\}=\left[\frac{d}{d t} \operatorname{Tr}\left\{\mathcal{T}^{t}(\rho)\right\}\right]_{t=0}=0
$$

implying

$$
0 \leq \operatorname{Tr}\left\{\mathcal{L}_{+}(\rho)\right\}=\operatorname{Tr}\{\mathcal{M}(\rho)\} \leq \operatorname{Tr}\{|\mathcal{M}(\rho)|\}=\|\mathcal{M}(\rho)\|_{1}, \quad \forall \rho \in \mathscr{D}_{0}, \quad \rho \geq 0 .
$$

In order to take also non-positive matrices into account we look at general matrix units.

10 LEMMA. For $(\phi, \psi) \subset \mathscr{D}(M)$ we have

$$
\left\|\mathcal{L}_{+}(|\phi\rangle\langle\psi|)\right\|_{1} \leq\left(\left\|\mathcal{L}_{+}(|\phi\rangle\langle\phi|)\right\|_{1}\right)^{1 / 2} \cdot\left(\left\|\mathcal{L}_{+}(|\psi\rangle\langle\psi|)\right\|_{1}\right)^{1 / 2} .
$$

Proof. We use generalized Kraus operators, see Section 6. In case their number is infinite we shall in the following first consider summations over finite $k \in$ $\{0, \ldots, K\}$ and then in the end look at $K \rightarrow \infty$. Moreover, the triangle inequality for the trace-norm and the Schwarz inequality for the inner product in $\ell^{2}$ shall be used. We have

$$
\begin{aligned}
& \left\|\mathcal{L}_{+}(|\phi\rangle\langle\psi|)\right\|_{1}=\| \sum_{k}\left|L_{k} \phi\right\rangle\left\langle L_{k} \psi\left|\left\|_{1} \leq \sum_{k}\right\|\right| L_{k} \phi\right\rangle\left\langle L_{k} \psi\right|\left\|_{1}=\sum_{k}\right\| L_{k} \phi\|\cdot\| L_{k} \psi \| \\
\leq & \left(\sum_{k}\left\|L_{k} \phi\right\|^{2}\right)^{1 / 2} \cdot\left(\sum_{k}\left\|L_{k} \psi\right\|^{2}\right)^{1 / 2}=\left(\left\|\mathcal{L}_{+}(|\phi\rangle\langle\phi|)\right\|_{1}\right)^{1 / 2} \cdot\left(\left\|\mathcal{L}_{+}(|\psi\rangle\langle\psi|)\right\|_{1}\right)^{1 / 2} .
\end{aligned}
$$

For a general matrix unit we establish approximations.

11 LEMMA. Consider two pairs, $\{(\phi, \psi),(e, f)\} \subset \mathscr{D}(M)$ which lie close to each other in the graph of $M$,

$$
(\|\phi-e\|,\|M \phi-M e\|,\|\psi-f\|,\|M \psi-M f\|) \subset[0, \varepsilon] .
$$

Then for actions of super operators the following approximations hold

$$
\|\mathcal{M}(|\phi\rangle\langle\psi|)-\mathcal{M}(|e\rangle\langle f|)\|_{1} \leq \varepsilon \cdot(\|\phi\|+\|M \phi\|+\|\psi\|+\|M \psi\|+2 \varepsilon),
$$

and

$$
\left\|\mathcal{L}_{+}(|\phi\rangle\langle\psi|)-\mathcal{L}_{+}(|e\rangle\langle f|)\right\|_{1} \leq \varepsilon \cdot(\|\phi\|+\|M \phi\|+\|\psi\|+\|M \psi\|+2 \varepsilon) .
$$


Proof.

$$
\begin{aligned}
& \|\mathcal{M}(|\phi\rangle\langle\psi|)-\mathcal{M}(|e\rangle\langle f|)\|_{1}=\|\mathcal{M}(|\phi-e\rangle\langle\psi|)+\mathcal{M}(|e\rangle\langle\psi-f|)\|_{1} \\
\leq & \|M \phi-M e\| \cdot\|\psi\|+\|\phi-e\| \cdot\|M \psi\|+\|M e\| \cdot\|\psi-f\|+\|e\| \cdot\|M \psi-M f\| \\
\leq & \varepsilon \cdot(\|\psi\|+\|M \psi\|+\|\phi\|+\varepsilon+\|M \phi\|+\varepsilon) .
\end{aligned}
$$

For the action of $\mathcal{L}_{+}$we use Lemma 10 and domination by $\mathcal{M}$ when acting on positive $\rho$. Therefore, we have

$$
\begin{aligned}
& \left\|\mathcal{L}_{+}(|\phi\rangle\langle\psi|)-\mathcal{L}_{+}(|e\rangle\langle f|)\right\|_{1}=\left\|\mathcal{L}_{+}(|\phi-e\rangle\langle\psi|)+\mathcal{L}_{+}(|e\rangle\langle\psi-f|)\right\|_{1} \\
\leq \quad & \left(\left\|\mathcal{L}_{+}(|\phi-e\rangle\langle\phi-e|)\right\|_{1}\right)^{1 / 2} \cdot\left(\left\|\mathcal{L}_{+}(|\psi\rangle\langle\psi|)\right\|_{1}\right)^{1 / 2} \\
& +\left(\left\|\mathcal{L}_{+}(|e\rangle\langle e|)\right\|_{1}\right)^{1 / 2} \cdot\left(\left\|\mathcal{L}_{+}(|\psi-f\rangle\langle\psi-f|)\right\|_{1}\right)^{1 / 2} \\
\leq \quad & \left(\|\mathcal{M}(|\phi-e\rangle\langle\phi-e|)\|_{1}\right)^{1 / 2} \cdot\left(\|\mathcal{M}(|\psi\rangle\langle\psi|)\|_{1}\right)^{1 / 2} \\
& +\left(\|\mathcal{M}(|e\rangle\langle e|)\|_{1}\right)^{1 / 2} \cdot\left(\|\mathcal{M}(|\psi-f\rangle\langle\psi-f|)\|_{1}\right)^{1 / 2} \\
\leq \quad & 2 \varepsilon \cdot\left[(\|M \psi\| \cdot\|\psi\|)^{1 / 2}+(\|M e\| \cdot\|e\|)^{1 / 2}\right] \\
\leq & \varepsilon \cdot(\|M \psi\|+\|\psi\|+\|M \phi\|+\|\phi\|+2 \varepsilon) .
\end{aligned}
$$

Here we used the inequality between the geometric and arithmetic mean to transform the penultimate line.

12 PROPOSITION. For a generator $\mathcal{L}$ in generalized standard form there exists a basis $\left\{e_{k}\right\}$ such that $\mathscr{D}_{e}$ is a core for $M$, and $\mathscr{D}_{e}^{2}$ is a core for $\mathcal{L}$.

Proof. The graph $\{(\phi, M \phi), \phi \in \mathscr{D}(M)\}$ of the closed operator $M$ forms a complete Hilbert space with the graph norm $\|(\phi, M \phi)\|^{2}=\|\phi\|^{2}+\|M \phi\|^{2}$. In this Hilbert space there exists a basis $\left\{\left(\phi_{k}, M \phi_{k}\right)\right\}$. Since $\mathscr{D}(M)$ is dense in $\mathscr{H}$, the $\left\{\phi_{k}\right\}$ make a total set, and by the Gram Schmidt orthogonalization one can construct out of them a basis $\left\{e_{k}\right\}$ of $\mathscr{H}$. Both the Gram Schmidt procedure and its reverse, representing each $\phi_{k}$ by the $\left\{e_{k}\right\}$, involve only finite linear combinations, so the subspace $\mathscr{D}_{e}$ is a core for $M$.

Now a general $\rho \in \mathscr{D}_{0}$ is of finite rank and thus a finite sum of matrix units,

$$
\rho=\sum_{n=1}^{N}\left|\phi_{n}\right\rangle\left\langle\psi_{n}\right| .
$$

For any $\varepsilon>0$ there are vectors $e_{n}, f_{n}$ in $\mathscr{D}_{e}$ close to $\phi_{n}, \psi_{n}$ in graph norm. Due to these approximations in graph norm Lemma 11 can be applied. Combining both inequalities stated there, yields

$$
\left\|\mathcal{L}\left(\left|\phi_{n}\right\rangle\left\langle\psi_{n}\right|\right)-\mathcal{L}\left(\left|e_{n}\right\rangle\left\langle f_{n}\right|\right)\right\|_{1} \leq 2 \varepsilon \cdot\left(\left\|\phi_{n}\right\|+\left\|M \phi_{n}\right\|+\left\|\psi_{n}\right\|+\left\|M \psi_{n}\right\|+2 \varepsilon\right) .
$$

Then, by the triangle inequality and by summation of all these inequalities, we arrive at

$$
\begin{aligned}
& \left\|\mathcal{L}(\rho)-\mathcal{L}\left(\sum_{n}\left|e_{n}\right\rangle\left\langle f_{n}\right|\right)\right\|_{1} \leq \sum_{n}\left\|\mathcal{L}\left(\left|\phi_{n}\right\rangle\left\langle\psi_{n}\right|\right)-\mathcal{L}\left(\left|e_{n}\right\rangle\left\langle f_{n}\right|\right)\right\|_{1} \\
& \leq N \cdot \varepsilon \cdot\left(\max _{n}\left(\left\|\phi_{n}\right\|+\left\|M \phi_{n}\right\|+\left\|\psi_{n}\right\|+\left\|M \psi_{n}\right\|\right)+2 \varepsilon\right) .
\end{aligned}
$$


Now $\mathscr{D}(\mathcal{L})$ is the closure of the set of operators with finite rank in $\mathscr{D}(\mathcal{L})$, so it follows that $\mathscr{D}_{e}^{2}$ is a core for $\mathcal{L}$.

\section{Discussion}

QDS can be analyzed on three levels: the underlying Hilbert space, the space of Hilbert space operators, and the "super-operators" acting on density operators. On the "highest level", the level of super-operators, we refer to general abstract theorems concerning strongly continuous semigroups in Banach spaces. The existence of a generator $\mathcal{L}$, under the condition that $\mathcal{T}^{t}$ is strongly continuous and trace preserving, is the presupposition and basis for all our definitions, propositions and theorems. On the intermediate level we discuss the form in which the generator can be represented using operators on the Hilbert space. This problem is, as is shown in this paper, related to the appearance of the QDS on the lowest level. On this level we may see, in principle, the evolution equation as a set of coupled differential equations for matrix elements in certain bases of $\mathscr{H}$.

What is new in our presentation, in comparison to Davies' definition of a "Standard Form"? We use $M^{\dagger}$ and $L^{\dagger}$ at places where Davies puts - formally the adjoint operators. In the present context, the adjoint $M^{*}$ exists with a dense domain of definition, but its range is in general not large enough; the operators $L_{k}$ are in general not closable, and the $L_{k}^{*}$ not densely defined. These features can be explicitly seen in the examples illustrated in Section 5. Looking closely into Davies' papers, one may observe that he just writes $M^{*}$ and $L^{*}$, without really using these adjoint operators, when giving a precise sense to the formal notations. Being honest, we admit that our "generalized standard form" is therefore not really new. But his kind of notation may lead to some misunderstandings, which we try to avoid. To characterize $M^{\dagger}$ and $L^{\dagger}$ more precisely, one may note that these operators map $\mathscr{H}$ into the dual space of $\mathscr{H}_{M}$, in the Gelfand triple $\mathscr{H}_{M} \subset$ $\mathscr{H} \subset \mathscr{H}_{M}^{*}$, where $\mathscr{H}_{M}$ is the domain of definition of $M$ equipped with the norm $\|\phi\|_{M},\|\phi\|_{M}^{2}=\|\phi\|^{2}+\|M \phi\|^{2}$.

Remark on the notation: Our notation indicates that $\mathcal{L}(\rho)$ denotes a trace class operator defined on the whole Hilbert space $\mathscr{H}$. The restriction onto a domain concerns the set of density matrices, not of Hilbert space vectors. We choose nevertheless to write $\mathcal{L}$ in a way which is similar to Davies' expression of a standard form, and which looks like a formula in matrix analysis. This is a matter of taste; if one doesn't like the $M^{\dagger}$ and the $L^{\dagger}$, one can write, instead of (3) and (4)

$$
\mathcal{L}(\rho)=\sum_{k} L_{k}\left(L_{k} \rho^{*}\right)^{*}-M \cdot \rho-\left(M \cdot \rho^{*}\right)^{*}
$$

with

$$
\sum_{k}\left\|L_{k} \phi\right\|^{2}=\langle\phi \mid M \phi\rangle+\langle M \phi \mid \phi\rangle, \quad \forall \phi \in \mathscr{D}(M) .
$$


Remark on the domains: To construct the operators appearing in the generalized standard form it is not sufficient to assume that $\mathscr{D}_{e}^{2}$ is contained in the domain of definition of $\mathcal{L}$. As a well-known counter example consider an induced unitary evolution $\mathcal{T}^{t}(\rho)=U_{t} \rho U_{t}^{*}$, where the generator of the group $U_{t}$ is a Laplacian on a finite interval $[a, b]$ with Dirichlet boundary conditions. With a basis $\left\{e_{k}\right\}$, where the limits $x \rightarrow a$ and $x \rightarrow b$ of each function $e_{k}(x)$ and of its derivative is 0 , the matrix elements give no information on boundary conditions. They define an hermitian operator with nonzero defect indices.

From the point of view of a functional analyst some important problems still demand further studies: One is the question under which conditions a strongly continuous QDS is matrix normal in an appropriate basis. Another problem is how to reverse the implications, to give a functional analytic characterization of the generators, appearing in the form given in Definition 1, without the a priori assumption that the semigroup is trace preserving. This problem appears in the literature, starting essentially with [C91], continuing with many more investigations, e.g. [CF98, [FPM12], as finding conditions for the generated semigroup to be "conservative". Last but not least, one would like to have a "standard non-standard form" for generators which are not matrix normal. We have examples, one from Davies and one from Holevo, of such non-standard generators, [D77, K95, H96]. Both are built as extensions of generators in standard form, but generating semigroups which do not preserve the trace. The partition $\mathcal{L}=\mathcal{L}_{+}-\mathcal{M}$, defined here with Equations (3, 4,6]15) is extended to $\mathcal{L}=\mathcal{L}_{n}+\mathcal{L}_{+}-\mathcal{M}$, including a non-standard completely positive map $\mathcal{L}_{n}$ which is not to be represented with generalized Kraus operators. Is this the ultimate most general form? A question that still needs to be answered.

\section{Examples}

\subsection{Translation, followed by drop out}

The following simple example shows all the special features of the present analysis.

(A similar example is presented in [A02, sect. 2)

Consider the Hilbert space

$$
\mathscr{H}=\mathscr{L}^{2}\left(\mathbb{R}_{+}\right) \oplus \mathbb{C}=\left\{\left(\begin{array}{c}
\psi(x) \\
a
\end{array}\right)\right\}
$$

and define

$$
|\omega\rangle:=\left(\begin{array}{l}
0 \\
1
\end{array}\right), \quad \Omega:=|\omega\rangle\langle\omega| .
$$

Moreover,

$$
\mathcal{T}^{t}(\rho)=\operatorname{Tr}\left(P_{t} \rho\right) \Omega+S_{t} \rho S_{t}^{*}
$$


with

$$
S_{t}|\omega\rangle=|\omega\rangle, \quad\left(S_{t} \psi\right)(x)=\psi(x+t), \quad P_{t}|\omega\rangle=0, \quad\left(P_{t} \psi\right)(x)=\chi_{[0, t]}(x) \psi(x) .
$$

The generator is given by

$$
\mathcal{L}(\rho)=L \rho L^{\dagger}+\partial \rho+\rho \partial^{\dagger}
$$

where $\partial|\omega\rangle=0$ and $(\partial \psi)(x)=\psi^{\prime}(x)$ without boundary conditions, and

$$
L\left(\begin{array}{c}
\psi(x) \\
a
\end{array}\right)=\left(\begin{array}{c}
0 \\
\psi(0)
\end{array}\right), \quad \mathscr{D}(L) \supset \mathscr{D}(\partial)
$$

Note that $L$ - formally $L=|\omega\rangle\langle\delta(x)|$ - is not closable, so $L^{*}$ is not a densely defined operator. As in Equation (2),$L^{\dagger}$ is a generalized operator, a linear map from $\mathscr{H}$, the space of ket-vectors, to a space of unbounded linear functionals on the Hilbert space of bra-vectors. Formally $L^{\dagger}=|\delta(x)\rangle\langle\omega|$.

The dual semigroup acts as

$$
\mathcal{T}^{t *}(A)=\langle\omega|A| \omega\rangle P_{t}+S_{t}^{*} A S_{t}
$$

It does not map $\mathscr{C}(\mathscr{H})$, the space of compact operators, into $\mathscr{C}(\mathscr{H})$. It, therefore, does not fulfill the condition stated in Theorems 3.4 and 4.1 in [D77b], a requirement necessary in Davies' approach to prove that the generator can appear in standard form. It is not even strongly continuous and has no generator in the usual sense, namely as an operator mapping $\mathscr{B}(\mathscr{H})$ to $\mathscr{B}(\mathscr{H})$.

One could define a generalized "standard" form for the dual generator, mapping $\mathscr{B}(\mathscr{H})$ to the set of quadratic forms $\mathfrak{Q}(\mathscr{H})$, by

$$
\mathcal{L}^{\prime}(A)=L^{\dagger} A L+\partial^{\dagger} A+A \partial .
$$

It involves a completely positive mapping of $A$ to the quadratic form $L^{\dagger} A L$. A general theory of such extended Kraus operators is given in the Appendix in Definition 13 and Theorem 14. Another characterization has been stated by Holevo in [H97]. He considered the generator as a bilinear functional, a "form", acting onto a pair which consists of an observable and a density matrix.

We note that this example is not far from representing a physical process as described in the following subsection.

\subsection{Quantum reflection with sticking probability}

We present a simple model for a process observed in modern physics, see [ZB83, BD06. Consider a hydrogen atom that moves freely in half space and is reflected from the surface of ultracold helium, the plane which is the boundary to the other half space. With some probability, however, it remains bounded to this surface and creates there a "ripplon". 
To describe this situation in a mathematical model, we consider the Fock space $\mathcal{F}$ of ripplons, the space $1 \mathscr{L}^{2}\left(\mathbb{R}^{2}, d x d y\right)$ of wave functions for the hydrogen bounded to the surface, and the space of wave functions in the half space, $\mathscr{L}^{2}\left(\mathbb{R}^{2}, d x d y\right) \otimes \mathscr{L}^{2}\left(\mathbb{R}_{+}, d z\right)$. The total Hilbert space, therefore, is

$$
\begin{aligned}
\mathscr{H} & =\mathcal{F} \otimes\left[\mathscr{L}^{2}\left(\mathbb{R}^{2}, d x d y\right) \oplus \mathscr{L}^{2}\left(\mathbb{R}^{2}, d x d y\right) \otimes \mathscr{L}^{2}\left(\mathbb{R}_{+}, d z\right)\right] \\
& =\mathcal{F} \otimes \mathscr{L}^{2}\left(\mathbb{R}^{2}, d x d y\right) \otimes\left(\mathbb{C} \oplus \mathscr{L}^{2}\left(\mathbb{R}_{+}, d z\right)\right) .
\end{aligned}
$$

The free movement yields the Hamiltonian, here forming the imaginary part of the "mover-and-contractor" $M$,

$$
M=i\left(H_{R}+H_{2}+H_{z}\right) .
$$

$H_{R}$ is the self-adjoint Hamiltonian for the free ripplons. $H_{2}$ is also self-adjoint and is the negative Laplacian on the plane, representing both the free movement of the hydrogen atom when it is bounded to the surface, and the parallel part of free movement in the half plane. The evolution in the direction orthogonal to the boundary is modeled by $H_{z}=-\frac{d^{2}}{d z^{2}}$ with boundary conditions $\psi^{\prime}(0)=w \psi(0)$, $w \in \mathbb{C}, \operatorname{Im}(w)>0$. This part is not self-adjoint, so $M+M^{\dagger}=i\left(H_{z}-H_{z}^{\dagger}\right)$. Moreover, the imaginary part of $w$ is related to the probability of sticking. The transfer of the hydrogen atom into the layer at the surface is modeled by $L_{z}$ acting on $\mathbb{C} \oplus \mathscr{L}^{2}\left(\mathbb{R}_{+}, d z\right)$ as

$$
L_{z}|\psi\rangle=(2 \operatorname{Im}(w))^{1 / 2}|\omega\rangle \psi(0)
$$

and $L_{z}|\omega\rangle=0$, where $|\omega\rangle$ is a unit vector in the Hilbert space $\mathbb{C}$. To complete the physics, there is an isometry from $\mathcal{F} \otimes \mathscr{L}^{2}\left(\mathbb{R}^{2}\right) \otimes \mathbb{C}$ to $\mathcal{F} \otimes \mathscr{L}^{2}\left(\mathbb{R}^{2}\right) \otimes \mathbb{C}$, namely the scattering matrix $S$. It creates a ripplon with angular momentum $\kappa$ and reduces the momentum of the sticking hydrogen from $k$ to $k-\kappa$. These vectors of momenta are all in the plane and $\kappa$ has the same direction as $k$. The absolute value of $\kappa$ depends on $|k|$ and is consistent with the conservation of total energy. On the total Hilbert space the generalized Kraus operator $L=S \cdot L_{z}$ is then acting.

From the perspective of mathematical physics, one would like to improve this model and also to derive it from Schrödinger quantum mechanics. Our conjecture is that this task can be fulfilled by using a strong coupling limit.

\footnotetext{
${ }^{1}$ What we denote here by $\mathscr{L}^{2}$ is the usual Hilbert space $L^{2}$, since the letter $L$ is reserved for operators.
} 


\section{Appendix: Generalized Kraus operators for unbounded completely positive maps}

\subsection{Generalization of "completely positive map"}

K. Kraus, [K71] gave a representation of the Stinespring theorem in the form

$$
\mathcal{Q}(W)=\sum_{k} K_{k} \cdot W \cdot K_{k}^{*}
$$

where $\mathcal{Q}$ is an "operation", a completely positive bounded map from $\mathfrak{T}(\mathscr{H})$ to $\mathfrak{T}(\mathscr{H})$. It has been observed several times, e.g. in [SG05], that in case of a finite dimensional Hilbert space $\mathscr{H}$ this representation can be deduced from the Choi-Jamiolkowski dualism of maps and states.

We give here a generalization to infinite dimensions and densely defined unbounded completely positive maps. Moreover, we consider the case, where the target of the map is not a space of operators but the set of quadratic forms, $\mathfrak{Q}(\mathscr{H})$.

\section{DEFINITION. Generalized Operators:}

Consider two bases, $\left\{e_{n}\right\}$ and $\left\{f_{n}\right\}$ in the Hilbert space of infinite dimension. The operators $K$, used in the following, are linear mappings from $\mathscr{D}_{e}$ into the algebraic dual of $\mathscr{D}_{f}$. Their adjoints, $K^{\dagger}$, are operators from $\mathscr{D}_{f}$ into the algebraic dual of $\mathscr{D}_{e}$, related to $K$ by

$$
\left\langle e\left|K^{\dagger}\right| f\right\rangle=\langle f|K| e\rangle^{*}
$$

for $e \in \mathscr{D}_{e}, f \in \mathscr{D}_{f}$.

In the sense of Holevo's investigations these operators can also be considered as bilinear forms on $\operatorname{lin}\{|f\rangle\langle e|\}$ and $\operatorname{lin}\{|e\rangle\langle f|\}$ respectively. In another sense one may characterize $M^{\dagger}$ and $L^{\dagger}$ more precisely as acting in the Gelfand triple $\mathscr{H}_{M} \subset \mathscr{H} \subset \mathscr{H}_{M}^{*}$. These operators map there $\mathscr{H}$ into the dual space of $\mathscr{H}_{M}$, which is the domain of definition of $M$ equipped with the norm $\|\phi\|_{M},\|\phi\|_{M}^{2}=$ $\|\phi\|^{2}+\|M \phi\|^{2}$.

\section{THEOREM. Generalized Kraus-Operators}

If $\mathcal{Q}$ is a completely positive map from $\mathscr{D}_{e}^{2}$ into $\mathfrak{Q}(\mathscr{H})$ with $\mathscr{D}_{f}$ as common form domain, there exists a countable set of "generalized Kraus operators" $K_{\alpha}$ such that

$$
\mathcal{Q}(\rho)=\sum_{\alpha} K_{\alpha} \rho K_{\alpha}^{\dagger}
$$

If, moreover, each quadratic form $\mathcal{Q}(\rho)$ can be associated with an operator $\breve{\mathcal{Q}}(\rho)$ in $\mathscr{B}(\mathscr{H})$, such that $\langle g|\mathcal{Q}(\rho)| f\rangle=\langle g \mid \breve{\mathcal{Q}}(\rho) f\rangle$ holds for all $f \in \mathscr{D}_{f}$ and for all $g \in \mathscr{D}_{f}$, each $K_{\alpha}$ is an operator mapping $\mathscr{D}_{e}$ into $\mathscr{H}$.

Note that there exist moreover several considerations of "Completely Positive Maps" in a different context, see e.g. [CTU11, P12]. 


\subsection{Proof of Theorem 14 and Corollaries on special cases}

We consider matrix units $E_{i, j}=\left|e_{i}\right\rangle\left\langle e_{j}\right|$, so that

$$
Q_{i, j ; k, \ell}=\left\langle f_{i}\left|\mathcal{Q}\left(E_{k, \ell}\right)\right| f_{j}\right\rangle
$$

Since the Choi matrix $E=\sum_{i, j} E_{i, j} \otimes E_{i, j}$ in infinite dimensions is a quadratic form [H11], not an operator, we use the approximating increasing sequence of bounded positive operators on $\mathscr{H} \otimes \mathscr{H}$

$$
E_{N}=\sum_{i, j}^{N} E_{i, j} \otimes E_{i, j}=\left|\theta_{N}\right\rangle\left\langle\theta_{N}\right|, \quad \text { with } \quad\left|\theta_{N}\right\rangle=\sum_{i}^{N}\left|e_{i} \otimes e_{i}\right\rangle .
$$

Next, we make use of complete positivity of $\mathcal{Q}$ and look at the map $\hat{\mathcal{Q}}=\mathcal{Q} \otimes \mathbb{1}$, which preserves positivity. It is, therefore, possible to form the positive square root of $\hat{\mathcal{Q}}\left(E_{N}\right)$ and define vectors

$$
\Psi_{N ; i, k}=\sqrt{\hat{\mathcal{Q}}\left(E_{N}\right)}\left|f_{i} \otimes e_{k}\right\rangle
$$

These vectors are unnormalized and change with $N$. But observe that, for $N \geq k$

$$
\left\|\Psi_{N ; i, k}\right\|^{2}=\left\langle f_{i} \otimes e_{k}\left|\hat{\mathcal{Q}}\left(E_{N}\right)\right| f_{i} \otimes e_{k}\right\rangle=\left\langle f_{i}\left|\mathcal{Q}\left(E_{k, k}\right)\right| f_{i}\right\rangle \leq \infty
$$

and, for $N \geq k$ and $N \geq \ell$

$$
\begin{aligned}
\left\langle\Psi_{N ; i, k} \mid \Psi_{N ; j, \ell}\right\rangle=\left\langle f_{i} \otimes e_{k}\left|\hat{\mathcal{Q}}\left(E_{N}\right)\right| f_{j} \otimes e_{\ell}\right\rangle & = \\
=\left\langle f_{i} \otimes e_{k}\left|\mathcal{Q}\left(E_{k, \ell}\right) \otimes E_{k, \ell}\right| f_{j} \otimes e_{\ell}\right\rangle & =\left\langle f_{i}\left|\mathcal{Q}\left(E_{k, \ell}\right)\right| f_{j}\right\rangle .
\end{aligned}
$$

The vectors $\Psi$ will, most probably, go weakly to zero, as $N \rightarrow \infty$, but their norms and inner products are bounded, increasing in $N$ and remaining constant for large $N$. So the internal "geometry" of the rhomboid which they form stays constant and can be represented with a fixed set of vectors. This can be done by mimicking the Gram-Schmidt procedure, generating an orthonormal set of vectors out of the $\Psi$-vectors for any finite $N$, and mapping them onto a fixed basis.

\section{PROPOSITION. Construction of the $N$-independent vectors}

$A$ set of vectors $\Phi_{i, k} \in \mathscr{H} \otimes \mathscr{H}$ can be constructed independently of $N$, such that

$$
\left\langle\Phi_{i, k} \mid \Phi_{j, \ell}\right\rangle=\left\langle\Psi_{N ; i, k} \mid \Psi_{N ; j, \ell}\right\rangle, \quad \forall N \geq \max \{i, j, k, \ell\} .
$$

Proof. We first choose the ordering of indices for the basis $e_{k}$ in such a way, that $\Psi_{N ; 0,0} \neq 0$ and then define some order relation for the index-pairs $(i, k)$. More 
precisely, we set $n=n(i, k):=\max \{i, k\}, \alpha=\alpha(i, k):=n^{2}-n+i-k$ and denote $\Phi_{\alpha}=\Phi_{i, k}$.

Consider now an arbitrary basis $b_{\alpha}$ and make the Ansatz:

$$
\Phi_{\alpha}=\sum_{\beta \leq \alpha} \gamma_{\alpha, \beta} b_{\beta}
$$

Note that $\left\|\Phi_{0}\right\|^{2} \neq 0$, hence

$$
\gamma_{0,0}:=\left\|\Phi_{0}\right\|>0
$$

Next, we proceed with nested inductions on $\alpha$ and $\beta$ with $\beta \leq \alpha$. Having determined all $\gamma_{\alpha-1, \beta}$ for fixed $\alpha-1$, we go over to fixed $\alpha$ and determine the sequence $\gamma_{\alpha, \beta}$, in increasing order of $\beta$. To this end, we solve Equation (26) and take for $\beta<\alpha$ the expansion

$$
\left\langle\Phi_{\beta} \mid \Phi_{\alpha}\right\rangle=\left[\sum_{\beta^{\prime}<\beta} \gamma_{\beta, \beta^{\prime}}^{*} \gamma_{\alpha, \beta^{\prime}}\right]+\gamma_{\beta, \beta}^{*} \gamma_{\alpha, \beta},
$$

into account, which determines $\gamma_{\alpha, \beta}$ in case $\gamma_{\beta, \beta} \neq 0$.

If $\gamma_{\beta, \beta}=0$, the vector $\Phi_{\beta}$ is a linear combination of $\Phi_{\beta^{\prime}}$ with $\beta^{\prime}<\beta$. This already guarantees the validity of Equation (26). In such a case we reject any use of $b_{\beta}$ and set $\gamma_{\alpha, \beta}=0$ for each $\alpha>\beta$, to make sure that the induction on $\beta$ can be finished at $\beta=\alpha$. This procedure guarantees, at the end of induction on $\beta$, the solvability of

$$
\left\langle\Phi_{\alpha} \mid \Phi_{\alpha}\right\rangle=\left[\sum_{\beta<\alpha}\left|\gamma_{\alpha, \beta}\right|^{2}\right]+\left|\gamma_{\alpha, \alpha}\right|^{2}
$$

enabling us to determine $\gamma_{\alpha, \alpha} \geq 0$ as the positive square root of $\left\|\Phi_{\alpha}\right\|^{2}-\sum\left|\gamma_{\alpha, \beta}\right|^{2}$.

One may represent now the super matrix elements by combining Equations (24, 25, 26)

$$
Q_{i, j ; k, \ell}=\left\langle\Phi_{i, k} \mid \Phi_{j, \ell}\right\rangle=\sum_{\alpha}\left\langle\Phi_{i, k} \mid \alpha\right\rangle\left\langle\alpha \mid \Phi_{j, \ell}\right\rangle
$$

where $|\alpha\rangle$ are some basis vectors in $\mathscr{H} \otimes \mathscr{H}$. We shall consider the basis $|\alpha\rangle=b_{\alpha}$ with $\alpha=\alpha(j, \ell)$, which was used in the proof of the Proposition 15. Note that, due to the Gram-Schmidt-like procedure used to construct the $\Phi$-vectors, there appears for each $\Phi_{i, k}$ only a finite set of $\alpha$ in Equation (28). So one can swap the two sums in Equation (29) following below.

Define now "Kraus-operators" $K_{\alpha}$ on $\mathscr{D}_{e}$ by their matrix-elements

$$
\left\langle f_{i}\left|K_{\alpha}\right| e_{k}\right\rangle:=\left\langle\Phi_{i, k} \mid \alpha\right\rangle
$$


Then with (27), we have for matrices $\rho$ which are finite linear combinations of $E_{k, \ell}$ that

$$
\begin{aligned}
{[\mathcal{Q}(\rho)]_{i, j} } & =\sum_{k, \ell} Q_{i, j ; k, \ell} \rho_{k, \ell}=\sum_{k, \ell} \sum_{\alpha}\left\langle\Phi_{i, k} \mid \alpha\right\rangle \rho_{k, \ell}\left\langle\alpha \mid \Phi_{j, \ell}\right\rangle=\sum_{\alpha} \sum_{k, \ell} \ldots=(29) \\
& =\sum_{\alpha}\left(K_{\alpha} \rho K_{\alpha}^{\dagger}\right)_{i, j}
\end{aligned}
$$

16 COROLLARY. If $\mathcal{Q}$ maps $\mathscr{D}_{e}^{2}$ into $\mathscr{B}(\mathscr{H})$, the Kraus Operators $K_{\alpha}$ map $\mathscr{D}_{\text {e }}$ into $\mathscr{H}$.

Proof. Consider $\phi \in \mathscr{D}_{e}$ and form $|\phi\rangle\langle\phi|$ which is in $\mathscr{D}_{e}^{2}$ and is mapped by $\mathcal{Q}$ to an operator with finite norm. For each $\psi \in \mathscr{D}_{f}$ which is dense in $\mathscr{H}$ we have

$$
\|\mathcal{Q}(|\phi\rangle\langle\phi|)\| \cdot\|\psi\|^{2} \geq\langle\psi|\mathcal{Q}(|\phi\rangle\langle\phi|)| \psi\rangle=\sum_{\alpha}\left\langle\psi\left|K_{\alpha}\right| \phi\right\rangle\left\langle\phi\left|K_{\alpha}^{\dagger}\right| \psi\right\rangle=\sum_{\alpha}\left|\left\langle\psi\left|K_{\alpha}\right| \phi\right\rangle\right|^{2} .
$$

So $\|\mathcal{Q}(|\phi\rangle\langle\phi|)\|$ gives a bound for the supremum over all normed $\psi$ and thus an upper bound for the square of the norm of each $K_{\alpha}|\phi\rangle$.

However, note that the $K_{\alpha}^{\dagger}$ are in general still "generalized" operators.

17 COROLLARY. For trace preserving maps $\mathcal{Q}$ the $K_{\alpha}$ can be extended to bounded operators in $\mathscr{B}(\mathscr{H})$, and $K_{\alpha}^{\dagger}=K_{\alpha}^{*}$.

Proof. For arbitrary positive $\rho$ the formula holds for a sequence of approximating positive $\rho_{N} \in \mathscr{D}_{e}^{2}$ with finite rank. Moreover, in the limit $N \rightarrow \infty$ we have bounded convergence.

Note that the preservation of trace by $\mathcal{Q}$ implies

$$
\sum_{i}\left\|\Phi_{i, k}\right\|^{2},=1, \quad \forall k \quad \Rightarrow \quad \sum_{i}\left\langle\Phi_{i, k} \mid \Phi_{i, \ell}\right\rangle=0, \quad \forall k, \ell
$$

therefore,

$$
\sum_{\alpha} K_{\alpha}^{\dagger} \cdot K_{\alpha}=\mathbb{1}, \quad \Rightarrow \quad\left\|K_{\alpha}\right\| \leq 1, \quad \forall \alpha
$$

\subsection{Acknowledgements}

We thank Franco Fagnola, who spotted an error in the first version and gave to us important hints on relations to existing papers.

One of us (S.A.) has been supported through the FWF-project Nr. P22929N16.

B.B. thanks V. Umanità and the organizing committee of the Workshop "Quantum Markov Semigroups: Decoherence and empirical estimates", Genova, 26-28 June 2013, for invitation to this marvelous workshop.

We thank also Jan Schlemmer for important hints on methods. 
Generators of Quantum Dynamical Semigroups

Dezember, 2013

\section{References}

[A02] W. Arveson: The domain algebra of a CP-semigroup. Pacific J, of Math. 203(1), $67-77$, (2002)

[BD06] Jean-Louis Basdevant, Jean Dalibard: The Quantum Mechanics Solver. Springer-Verlag Berlin Heidelberg, (2006)

[C91] A. M. Chebotarev: Necessary and Sufficient Conditions for Dynamical Semigroups to be Conservative. J. Soviet. Math. 56 (5), 2697 - 2719, (1991) Translated from Itogi Nauki i Tekhniki 36, 149 - 184, (1990)

[CF98] A. M. Chebotarev, Franco Fagnola: Sufficient conditions for conservativity of minimal quantum dynamical semigroups. Journal of functional analysis 153(2), $382-404$, (1998)

[CTU11] Giulio Chiribella, Alessandro Toigo, Veronica Umanità: Normal completely positive maps on the space of quantum operations. Quantum Probability and Related Topics: Proceedings of the 32nd Conference, 43 - 66, (2011) arXiv:1012.3197v3 [math-ph]

[D74] E. Brian Davies: Markovian Master Equations. Comm. Math. Phys. 39, 91 - 110, (1974)

[D76] E. Brian Davies: Markovian Master Equations II. Math. Ann. 219, $147-158,(1976)$

[D76b] E. Brian Davies: Quantum Theory of Open Systems. (Academic Press, New York) 1976

[D77] E. Brian Davies: Quantum Dynamical Semigroups and the Neutron Diffusion Equation. Rep. Math. Phys. 11, 169 - 188, (1977)

[D77b] E. Brian Davies: Generators of Dynamical Semigroups. J. Funct. Anal. 34, $421-432,(1979)$

[D80] E. Brian Davies: Uniqueness of the Standard Form of the Generator of a Quantum Dynamical Semigroup. Rep. Math. Phys. 17, 249 - 255, (1980)

[F99] Franco Fagnola: Quantum Markov Semigroups and Quantum Flows. Proyecciones, Journal of Math. 18(3), 1 - 144, (1999)

[FPM12] Franco Fagnola, L. Pantaleón Martínez: Are sufficient conditions for conservativity of minimal quantum semigroups necessary? Mathematical Notes, 91(6), 851 - 856, (2012) 
[FR06] Franco Fagnola and Rolando Rebolledo: Notes on the Qualitative Behaviour of Quantum Markov Semigroups. in: S.Attal, A.Joye, C.A.Pillet (Eds.): Open Quantum Systems III, Recent Developments. Springer-Verlag Berlin-Heidelberg, (2006)

[GKS76] Vittorio Gorini, Andrzej Kossakowsi, E.C.G.Sudarshan: Completely positive dynamical semigroups of N-level systems. J. Math. Phys. 17, $821-825,(1976)$

[H11] A. S. Holevo: Entropy Gain and the Choi Jamiolkowski Correspondence for Infinite-Dimensional Quantum Evolutions. Theor. Math. Phys. 166(1), 123 - 138, (2011)

[K95] A. S. Holevo (Kholevo): Excessive maps, "arrival times" and perturbation of dynamical semigroups. Izv. Ross. Akad. Nauk Ser. Mat. 59(6), 207 - 222, (1995); English transl. in Russian Acad. Sci. Izv. Math 59(6), $1311-1325,(1996)$

[H96] A. S. Holevo: There exists a non-standard dynamical semigroup on $\mathcal{L}(\mathscr{H})$. Comm. Moscow Math. Soc 51, 1206 - 07, (1996)

[H97] A. S. Holevo: Covariant Quantum Dynamical Semigroups: Unbounded Generators. arXiv:quant-ph/9701037

[K71] K. Kraus: General State Changes in Quantum Theory. Ann.Phys. (N.Y.) 64, $311-335,(1971)$

[L76] Gøran Lindblad: On the Generators of Quantum Dynamical Semigroups. Commun. Math. Phys. 48, 119 - 130, (1976)

[P12] Juha-Pekka Pellonpää: Completely positive maps on modules, instruments, extremality problems, and applications to physics. arXiv:1202.5905 [quant-ph], (2012)

[SG05] D. Salgado, J.L. Sánchez-Gómez and M. Ferrero: A Simple Proof of the Jamiotkowski Criterion for Complete Positivity of Linear Maps. Open Systems and Information Dynamics. 12(1), 56 - 64, (2005)

[ZB83] D. S. Zimmerman, A. J. Berlinsky: The sticking probability for hydrogen atoms on the surface of liquid ${ }^{4} \mathrm{He}$. Canadian Journal of Physics. 61(3), $508-513,(1983)$ 